\title{
Genetic testing for DADA2: How can we avoid missing patients?
}

\author{
Hafize Emine Sönmez ${ }^{1} \cdot$ Ezgi Deniz Batu ${ }^{1}$ Ekim Z. Taşkıran ${ }^{2,3} \cdot$ Mehmet Alikaşifoğlu $^{2,3} \cdot$ Yelda Bilginer $^{1,3} \cdot$ \\ Seza Özen ${ }^{1,3}$
}

Received: 27 May 2018 / Accepted: 18 July 2018 / Published online: 11 September 2018

(c) European Society of Human Genetics 2018

We read the article, 'A decision tree for the genetic diagnosis of deficiency of adenosine deaminase 2 (DADA2): a French reference centres experience' with great interest [1]. The authors have proposed a preliminary decision tree for DADA2 genetic testing. According to this algorithm, they suggested genetic analysis for DADA2 in presence of at least one item from each of three categories: signs of inflammation (fever and/or elevated C-reactive protein); signs of vasculitis (cutaneous and/or neurologic), and recurrent or chronic course (only for adults).

We applied this decision tree retrospectively to our pediatric patients (0-18 years) who were followed-up in Pediatric Rheumatology Outpatient Clinics of Hacettepe University between January 2010 and January 2018 with the diagnosis of DADA2 $(n=10)$ or polyarteritis nodosa (PAN) $(n=8)$. Three of these DADA2 patients were previously reported $[2,3]$. Sanger sequencing for CECR1 (cat eye syndrome chromosome region candidate 1) mutations had been performed in all of these patients before; nine DADA2 patients were homozygous and one was heterozygous for G47R mutation in CECR1. All PAN patients were negative for $C E C R 1$ mutations. All DADA2 patients but one met the prerequisites of the decision tree, while all PAN patients fulfilled the prerequisites. The DADA2 patient who did not meet the prerequisites, presented with only hematologic manifestation (aplastic anemia). And there was parental consanguinity in 9 DADA2 patients, while it was present in only two PAN patients. When we

Seza Özen

sezaozen@hacettepe.edu.tr

1 Department of Pediatrics, Division of Rheumatology, Hacettepe University Faculty of Medicine, Ankara, Turkey

2 Department of Medical Genetics, Hacettepe University Faculty of Medicine, Ankara, Turkey

3 Hacettepe University Center for Genomics and Rare Disease, Ankara, Turkey added the presence of parental consanguinity and hematologic manifestations to the prerequisites, all DADA2 patients fulfilled the prerequisites; however, none of the PAN patients except two of them met the new prerequisites. Both PAN patients who met the criteria for Sanger sequencing of CECRI had parental consanguinity and lymphopenia. Mutations in RAGl (recombinationactivating 1) were demonstrated with whole exome sequencing in one of these patients [4]. The genetic analysis is ongoing for the other patient.

DADA2 is a rare autoinflammatory disease with an expanding phenotype. Although it was first described in patients with PAN-like phenotype [3,5], recent reports have shown that DADA2 patients may present with isolated hematologic manifestations such as bone marrow failure or red cell aplasia [6-9]. Some of these patients lack signs of inflammation or vasculopathy $[8,10]$. Thus, it is important to include this feature as a prerequisite to the decision DADA2 testing.

DADA2 is an autosomal recessive disease. Clues that suggest autosomal recessive disorders such as parental consanguinity or family history of similar phenotype could be important to differentiate DADA2 from PAN. Thus, this may be added to the decision tree, as well.

In conclusion, modifying the prerequisites in the decision tree for DADA2 by adding hematologic manifestations and parental consanguinity will prevent us from overlooking DADA2 patients with atypical presentations.

\section{Compliance with ethical standards}

Conflict of interest The authors declare that they have no conflict of interest.

\section{References}

1. Rama M, Duflos C, Melki I, et al. A decision tree for the genetic diagnosis of deficiency of adenosine deaminase 2 (DADA2): a 
French reference centres experience. Eur J Hum Genet. 2018;26: 960-71.

2. Batu ED, Karadag O, Taskiran EZ, et al. A Case series of adenosine deaminase 2-deficient patients emphasizing treatment and genotype-phenotype correlations. J Rheumatol. 2015;42: 1532-4.

3. Zhou Q, Yang D, Ombrello AK, et al. Early-onset stroke and vasculopathy associated with mutations in ADA2. N Eng J Med. 2014;370:911-20.

4. Taskiran EZ, Sonmez HE, Ayvaz DC, et al. Hypomorphic RAG1 defect in a child presented with pulmonary hemorrhage and digital necrosis. Clin Immunol. 2018;187:92-4.

5. Navon Elkan P, Pierce SB, Segel R, et al. Mutant adenosine deaminase 2 in a polyarteritis nodosa vasculopathy. N Eng J Med. 2014;370:921-31.
6. Berkun Y, Segel R, Navon-Elkan P. Adenosine deaminase 2 deficiency: more than monogenic vasculitis. Isr Med Assoc J. 2017;19:435-7.

7. van Montfrans J, Zavialov A, Zhou Q. Mutant ADA2 in vasculopathies. N Eng. J Med. 2014;371:478.

8. Ben-Ami T, Revel-Vilk S, Brooks R, et al. Extending the clinical phenotype of adenosine deaminase 2 deficiency. J Pediatr. 2016;177:316-20.

9. Hashem H, Egler R, Dalal J. Refractory pure red cell aplasia manifesting as deficiency of adenosine deaminase 2. J Pediatr Hematol Oncol. 2017;39:293-6.

10. Schepp J, Proietti M, Frede N, et al. Screening of 181 patients with antibody deficiency for deficiency of adenosine deaminase 2 sheds new light on the disease in adulthood. Arthritis Rheumatol. 2017;69:1689-700.

\title{
Reply to Sönmez et al.
}

\author{
Mélanie Rama ${ }^{1}$ - Isabelle Touitou $\mathbb{1}^{1,2} \cdot$ Guillaume Sarrabay ${ }^{1,2}$
}

Received: 12 July 2018 / Accepted: 19 July 2018 / Published online: 11 September 2018

(c) European Society of Human Genetics 2018

We thank Sönmez et al. for their comment on our paper providing a provisional decision tree for the genetic diagnosis of deficiency of adenosine deaminase 2 (DADA2). This autosomal recessive disorder was primarily described as inflammatory vasculitis [1, 2]. More recently, other phenotypes have been described, such as pure haematological presentation (aplastic anaemia, erythroblastopenia, etc), without vascular or inflammation features [3-6].

Gene sequencing can be performed by either Sanger or NGS (Next Generation Sequencing) technique. In our original article, Rama et al. proposed a decision tree to help physicians prioritise molecular screening among SAID patients with possible DADA2 and benefit from a fast diagnosis with Sanger analysis [7]. Patients who do not meet the prerequisites have less probability to have DADA2. However, lack of some of the prerequisites does

Guillaume Sarrabay

guillaume.sarrabay@inserm.fr

1 Laboratory of Rare and Autoinflammatory Genetic Diseases and CEREMAIA, Montpellier University Hospital, Montpellier, France

2 Genetics \& Immunopathology of Inflammatory Osteoarticular Diseases, IRMB, Univ Montpellier, INSERM, Montpellier, France not exclude the diagnosis of DADA2, which can be made using NGS strategy.

Sönmez et al. have tested the proposed decision tree on ten genetically confirmed DADA2 patients and eight periarteritis nodosa (PAN) patients. All but one met the prerequisites: a true DADA2 patient with only haematological presentation who was not eligible for Sanger sequencing using the original tree. These authors pinpoint that atypical presentations such as aplastic anaemia are missed by our provisional decision tree. We concede that it might be relevant to implement the haematological features as an alternative prerequisite.

In the initial paper by Navon Elkan et al., CECRI/ADA2 was discovered in a cohort of PAN patients sequenced by WES, supporting that PAN and DADA2 may be undistinguishable based on clinical features only prior to genetic analysis [1]. Therefore, it is not surprising that all of their PAN patients meet the prerequisites. True PAN patients are not disadvantaged by having Sanger sequencing. The authors had a high prevalence of DADA2 consanguineous patients (9/10 patients). Consanguinity is common in the Turkish population, but rare in the European population and in our confirmed DADA2 patients (2/11 families) [7]. Hence, we believe that in most populations, consanguinity is not relevant as a prerequisite for Sanger analysis.

In conclusion, Sönmez et al. have highlighted the importance of the pure haematological presentation. They 
propose to implement this feature in the diagnostic decision, and we agree with that. However, consanguinity should be considered in specific populations, but cannot be universally used in our opinion.

\section{Compliance with ethical standards}

Conflict of interest The authors declare that they have no conflict of interest.

\section{References}

1. Navon Elkan P, Pierce SB, Segel R, Walsh T, Barash J, Padeh S, et al. Mutant adenosine deaminase 2 in a polyarteritis nodosa vasculopathy. N Engl J Med. 2014;370:921-31.
2. Zhou Q, Yang D, Ombrello AK, Zavialov AV, Toro C, Zavialov $\mathrm{AV}$, et al. Early-onset stroke and vasculopathy associated with mutations in ADA2. N Engl J Med. 2014;370:911-20.

3. Hashem H, Egler R, Dalal J. Refractory pure red cell aplasia manifesting as deficiency of adenosine deaminase 2. J Pediatr Hematol Oncol. 2017;39:e293-6.

4. van Montfrans J, Zavialov A, Zhou Q. Mutant ADA2 in vasculopathies. N Engl J Med. 2014;371:478.

5. Ben-Ami T, Revel-Vilk S, Brooks R, Shaag A, Hershfield MS, Kelly SJ, et al. Extending the clinical phenotype of adenosine deaminase 2 deficiency. J Pediatr. 2016;177:316-20.

6. Berkun Y, Segel R, Navon-Elkan P. Adenosine deaminase 2 deficiency: more than monogenic vasculitis. Isr Med Assoc J. 2017;19:435-7.

7. Rama M, Duflos C, Melki I, Bessis D, Bonhomme A, Martin H, et al. A decision tree for the genetic diagnosis of deficiency of adenosine deaminase 2 (DADA2): a French reference centres experience. Eur J Hum Genet. 2018;26:960-71. 